\title{
ENETS Consensus Guidelines for the Standards of Care in Neuroendocrine Tumors: Biochemical Markers
}

\author{
Dermot O'Toole Ashley Grossman $^{\mathrm{b}}$ David Gross ${ }^{c}$ Gianfranco Delle Fave ${ }^{\mathrm{d}}$ \\ Jaroslava Barkmanova ${ }^{\mathrm{e}}$ Juan $\mathrm{O}^{\prime}$ Connor ${ }^{\mathrm{f}}$ Ulrich-Frank Pape ${ }^{g}$ Ursula Plöckinger ${ }^{\mathrm{g}}$ \\ and all other Mallorca Consensus Conference participants \\ aDepartment of Gastroentereology and Clinical Medicine, St. James's Hospital and Trinity College, Dublin, Ireland; \\ ${ }^{b}$ St. Bartholomew, West Smithfield, London, UK; ' Department of Endocrinology and Metabolism, Hadassah University \\ Hospital, Jerusalem, Israel; dOspedale S. Andrea, Rome, Italy; e Department of Oncology, University Hospital, \\ Prague, Czech Republic; Instituto Alexander Fleming, Buenos Aires, Argentina; 9Department of Hepatology and \\ Gastroenterology, Campus Virchow-Klinikum, Charité-Universitätsmedizin Berlin, Berlin, Germany
}

\section{Standards for the Detection and Interpretation of 5-Hydroxyindole Acetic Acid}

\section{Background/Definition}

Neuroendocrine tumors (NETs) originating from the midgut may result in functional symptoms due to the secretion of various peptides and hormones and most notably 5-hydroxytryptamine (5-HT) or serotonin. This is a tryptophan-derived biogenic amine involved in smooth muscle contraction, blood pressure regulation and both peripheral and central nervous system neurotransmission. Approximately $2 \%$ of dietary tryptophan is converted into serotonin. Serotonin is synthesized and stored in enterochromaffin cells of the gastrointestinal tract $(80 \%$ of total body serotonin content), in dense granules of platelets (storage only) and in the serotoninergic neurons of the central nervous system. The urinary breakdown metabolite of serotonin is 5-hydroxyindole acetic acid (5HIAA) which is particularly useful in the diagnosis and follow-up of NETs with carcinoid syndrome. Serum measurements of serotonin are possible in these patients; however, large individual variation makes them unreliable for diagnosis and in follow-up. Universally, 5-HIAA is the most frequently performed assay in the clinical setting of the carcinoid syndrome.

\section{Performance of 5-HIAA in Diagnosis}

The overall sensitivity and specificity of urinary 5HIAA in the presence of the carcinoid syndrome is of the order of 70 and $90 \%$, respectively $[1,2]$. Midgut carcinoids are most liable to produce the carcinoid syndrome with 5-HIAA elevation, thus attesting to a high specificity $(>90 \%)$ in this setting. Fore- and hindgut NETs produce less serotonin than midgut tumors [1,3]. The sensitivity is lower in patients with midgut carcinoid tumors without the carcinoid syndrome. Urinary 5-HIAA levels may also depend on tumor volume and may be normal in patients with non-metastatic carcinoid tumors. Levels may be normal even in the presence of the carcinoid syndrome, particularly in subjects without diarrhoea; however, this is a rare event [4]. In functional midgut tumors, discriminating performances may vary depending on whether the cut-offs are high or low. Meijer et al. [1] demonstrated that a low level 5-HIAA cut-off value (2.8 $\mathrm{mmol} / \mathrm{mol}$ creatinine) yielded $68 \%$ sensitivity and $89 \%$ specificity, whereas a higher cut-off $(6.7 \mathrm{mmol} / \mathrm{mol} \mathrm{cre-}$ atinine) improved specificity to $98 \%$ at the expense of a lower sensitivity (52\%). Thus, in order to confidently exclude a carcinoid tumor, a low-level cut-off value may be preferred; to confirm the presence of a carcinoid tumor, a high-level cut-off value is better. Some patients with the

\section{KARGER}

Fax +4161306 1234

E-Mail karger@karger.ch

www.karger.com (c) 2009 S. Karger AG, Basel

0028-3835/09/0902-0194\$26.00/0

Accessible online at:

www.karger.com/nen
Dermot O'Toole

Department of Gastroenterology and Clinical Medicine

St. James's Hospital and Trinity College Dublin, James's Street

Dublin 8 (Ireland)

Tel. +353 1410 3815, Fax +3534410 3492, E-Mail otooled1@tcd.ie 
carcinoid syndrome excrete non-hydroxylated indole acids, not measured as 5-HIAA. There appears to be an inconstant correlation between 5-HIAA level and the clinical severity of the carcinoid syndrome; this may be related to a fluctuating release of serotonin from tumors such that the correlation may not be reliable. Recent data have examined 5-HIAA as a prognostic factor in these patients: while interesting data have emerged, the expert group felt that data have not confirmed 5-HIAA levels to be a consistently reliable prognostic factor in this disease. To illustrate this, two studies including 256 and 139 patients with midgut carcinoid tumors showed that while elevated 5-HIAA levels were predictive of poor outcome at univariate analysis, this did not remain significant at multivariate analysis $[5,6]$. In another study examining 76 patients, those with persistent moderately increased urinary 5-HIAA levels ( $\leq 20 \mathrm{mmol} / \mathrm{mol}$ creatinine) had a more favourable outcome compared to those with greatly elevated levels [7]. A further study in a mixed tumor group including 119 patients (53 of midgut origin) interestingly found high 5-HIAA to be an independent survival factor [8].

\section{Assays for 5-HIAA}

While several assays are available to measure 5-HIAA (thin-layer chromatography, enzyme immunoassay, gas chromatography, gas chromatography-mass spectrometry) [9-12], the use of high-performance liquid chromatography (HPLC) is most frequently employed. HPLC with electrochemical detection is currently recommended; however, automated assays [13] or those using mass spectrometry [12] may be available in some laboratories. Liquid chromatography tandem mass spectrometry assay appears to be a rapid assay with little necessity for repeat analyses because of chromatographic interference or dilutions [12]. A further automated method with online solid-phase extraction and HPLC and fluorometric detection has recently been shown to have increased precision and faster throughput compared to the manual solvent extraction method [14]. Whatever technique is used, it should be performed in accredited laboratories.

\section{Conditions for Optimal Assay (fig. 1)}

Urine should be collected and measured in plastic containers. Acid should be added to ensure sterility and hence stability. The sample should be stored in a refrigerator until analysis. All the urine passed over $24 \mathrm{~h}$ should be collected into the container, preferably by using a measuring jug. Collecting should be started at a defined time point following urination, and after that urine should be
- On day 1 , urinate into the container upon rising in the morning.

- Collect all subsequent urine for the next $24 \mathrm{~h}$.

- On day 2 , urinate into the container upon rising in the morning.

- Keep the sample in the refrigerator or a cool place during the collection period. Upon completion, return it as instructed as soon as possible.

Some foods contain high levels of serotonin which may increase the levels of urinary 5-HIAA and consumption should be avoided 3 days prior to urine collection: plums, pineapples, bananas, eggplants, tomatoes, avocados, and walnuts.

Certain medications may also interfere with the assay. If on medications this should be discussed with your specialist physician who will give further advice.

Fig. 1. Procedure for collection of a 24-hour urine sample.

collected until the same time point the next day (a precise 24-hour collection). Written instructions should be handed out including food and medication precautions (fig. 1).

\section{Care in Interpreting 5-HIAA Levels}

Intraindividual variation of 5-HIAA is also possible and this variation may be high, therefore two consecutive 24-hour collections should be performed and the mean value of these two can be taken, especially when the collection is required for diagnosis. A single specimen may be sufficient for follow-up purposes. Certain co-morbidities or associated disorders may have effects on the concentration of 5-HIAA. Falsely low 5-HIAA levels may be encountered in patients with renal impairment and those on haemodialysis. In addition, 5-HIAA may be increased in untreated patients with malabsorption, who have increased urinary tryptophan metabolites. Such patients include those with gluten-sensitive enteropathy (coeliac disease), tropical sprue, Whipple disease, intestinal stasis and cystic fibrosis (chronic intestinal obstruction) [1, 15]; plasma 5-HT, but not urinary 5-HIAA, have been elevated in diarrhoea-predominant irritable bowel syndrome [16]. A small number of normal individuals may have elevated urinary 5-HIAA and therefore other objective findings should be used in conjunction with tumor marker analysis to support the diagnosis of a carcinoid [17]. The following food substances are rich in dietary tryptophan and therefore patients should abstain from these for 3 days prior to urinary collection: plums, pineapples, ba- 
nanas, eggplants (aubergines), tomatoes, avocados and walnuts $[2,18,19]$. Certain medications may increase or decrease 5-HIAA urinary levels as follows:

- Increased: acetanilide, phenacetin, glyceryl guaiacolate (found in many cough syrups), methocarbamol, and reserpine, cisplatin, fluorouracil, melphalan, rauwolfia.

- Decreased: chlorpromazine, heparin, imipramine, isoniazid, levodopa, monoamine oxidase inhibitors, methenamine, methyldopa, phenothiazines, promethazine, and tricyclic antidepressants, chlorophenylalanine, corticotrophin, guanfacine, imipramine, isocarboxazid, isoniazid, levodopa, MAO inhibitors, moclobemide, octreotide.

Patients are frequently treated with somatostatin analogues and these are known to decrease levels of 5-HIAA; where possible, assays for diagnostic purposes should be made in patients not on somatostatin analogues, while in the follow-up setting, comparisons should be performed in patients on stable or comparable doses.

\section{Insulinoma: 72-Hour Fast}

NETs secreting insulin are termed insulinomas and are almost exclusively intrapancreatic in nature. Excessive insulin secretion leading to hypoglycaemia usually results in a combination of neurologic (diplopia, blurred vision, confusion, abnormal behavior and amnesia, seizures, coma, etc.) and autonomic (sweating, weakness, hunger, tremor, nausea, feelings of warmth, anxiety, palpitations) symptoms. Symptoms are usually related to the degree of insulin-induced hypoglycaemia but may be non-specific. Hypoglycaemia-induced clinical signs are classically present in the early morning preprandial phase or may be exercise-induced. The diagnosis is suggested in the presence of: (1) symptoms of hypoglycaemia; (2) glucose $<2.2 \mathrm{mmol} / \mathrm{l}$ (40 mg/dl; others use a threshold of $<3$ $\mathrm{mmol} / \mathrm{l}, 50 \mathrm{mg} / \mathrm{dl}$ ), and (3) relief of symptoms with administration of glucose [20]. This is known as Whipple's triad. The 72-hour fast is the gold standard for diagnosing insulinoma and relates to the integrity of patients' endogenous suppression of insulin in the face of hypoglycaemia. The fast attests to autonomous insulin secretion and the failure of appropriate insulin suppression in the presence of hypoglycaemia. Factitious hypoglycaemia secondary to exogenous use of insulin is suspected on the finding of high (often very high) serum insulin in combination with suppression of C-peptide. Sulphonylureas and related insulin secretagogues result in a clinical pic- ture similar to patients with insulinoma and may be diagnosed by a positive drug screen [21]. An overall approach to diagnosing and managing insulinoma has been provided elsewhere in a recent consensus statement [22].

\section{Supervised 72-Hour Fast}

This test has been verified as the gold standard in establishing a biochemical diagnosis of insulinoma [23]. Patients should be hospitalized in a specialist unit experienced in performing the test. A 72-hour period is universally recognized as the most appropriate duration [22] although some groups have proposed a shorter fast of $48 \mathrm{~h}$ $[24,25]$. Symptoms appear within $12 \mathrm{~h}$ for one third of patients, $80 \%$ within $24 \mathrm{~h}, 90 \%$ with $48 \mathrm{~h}$ and approaching $100 \%$ within $72 \mathrm{~h}$ [26]. Absolute values of glucose and insulin are the most important variables and any measurable insulin is abnormal when blood glucose drops to 2.5 $\mathrm{mol} / \mathrm{l}(45 \mathrm{mg} / \mathrm{dl})$. Assays used for the determination of insulin, pro-insulin, C-peptide and $\beta$-hydroxybutyrate may vary but should be performed in accredited laboratories. Very occasionally, an insulinoma is only revealed by hypoglycaemia induced by a mixed meal rather than fasting.

\section{Patient Information Scheme}

A detailed description of the fast should be provided to all patients with an information card to help in symptom identification. Patients should stay off all foods except for plain water, black tea or coffee and essential medications (particularly hypoglycaemic agents, e.g., sulphonylureas).

\section{Procedure}

The timing of the 72-hour fast is not critical - some teams prefer to perform the test early in the week when staffing levels may be higher and avoiding prolonging the test into the weekend. An oral glucose tolerance or mixed meal test can be performed before the fast. The patient should be monitored in a supervised environment and fasting should be accompanied by an intravenous line.

- Absolute blood (venous) determinations should be performed at least 2-4 times per day and when the patient describes symptoms. The test interpretation should be made using laboratory blood glucose assays; bedside measurements can be used in the presence of clinical symptoms to determine if more definitive measurements should be made.

- Blood should also be drawn for insulin measurement concurrently with glucose estimations, and assay for insulin and C-peptide when the hypoglycaemia is confirmed. 
- $\beta$-Hydroxybutyrate (or urinary ketones) should be measured at the end of the test in order to confirm the validity of the fast. A low level of hydroxybutyrate in the presence of hypoglycaemia confirms inappropriate insulin or insulin-like hormone secretion.

- A urinary assay for sulphonylureas should be performed as a specific request.

- Not all drugs are detected, e.g. repaglinide [21]; false positive results may also occur, e.g. on paracetamol.

- The results need to be confirmed with the local laboratory.

\section{Definition of Hypoglycaemia}

The endpoint of the test is documented hypoglycaemia.

- Documented blood glucose levels $\leq 2.2 \mathrm{mmol} / \mathrm{l}$ ( $\leq 40$ $\mathrm{mg} / \mathrm{dl}$; according to some $<3 \mathrm{nmol} / \mathrm{l}, 50 \mathrm{mg} / \mathrm{dl}$; levels may depend on age and sex);

- Concomitant insulin levels $>6 \mu \mathrm{U} / \mathrm{l}(\geq 36 \mathrm{pmol} / \mathrm{l} ; \geq 3$ $\mu \mathrm{U} / \mathrm{l}$ by ICMA);

- A $\beta$-hydroxybutyrate level $\leq 2.7 \mathrm{mmol} / \mathrm{l}$ can be used as a surrogate marker to confirm the validity of the fast and inappropriate insulin suppression;

- A glucagon test immediately after 72-hour fasting in patients without definite results has also been recommended;

- Exercise test immediately after 72-hour fasting in patients without definitive results may be performed in a supervised setting;

- Use of a ratio of insulin to glucose to aid in the diagnosis is not recommended.

\section{Standards for the Diagnosis of a Gastrinoma: Secretin Test}

The diagnosis of Zollinger-Ellison syndrome (ZES) can be established by the demonstration of elevated fasting serum gastrin (FSG) in the presence of low gastric $\mathrm{pH}$. FSG alone is not adequate to make the diagnosis of ZES because hypergastrinaemia can be seen in patients with achlorhydria associated with chronic atrophic fundus gastritis (e.g., pernicious anaemia) and in other conditions with hyperchlorhydria (e.g., Helicobacter pylori infection, gastric outlet obstruction, renal failure, antral Gcell syndromes, short bowel syndrome, retained antrum). In addition, the use of chronic proton pump inhibitors (PPIs) leads to high FSG levels and therefore gastrin provocative tests are needed to establish the diagnosis of
ZES. Indeed, in a recent prospective analysis, up to twothirds of gastrinoma patients were found to have FSG values $<10$-fold normal [27]. The gold standard is the secretin test [27-31]. This hormone, when given intravenously provokes an increase in serum gastrin and secondarily in gastric acid secretion. The most reliable data concerning the secretin test have emanated from the National Institute of Health studies in patients with sporadic and multiple endocrine neoplasia type-I (MEN1)-associated gastrinomas [27-31]. Recent consensus guidelines have described the criteria used for establishing the diagnosis of gastrinoma [31]; however, according to the expert committee, acid output studies are available to only a limited number of groups (including those experts' groups). For the NIH group the secretin test was useful in diagnosing ZES regardless of the extent or locations of the tumor, the presence or absence of MEN1 or the level of FSG (less than or greater than $1,000 \mathrm{pg} / \mathrm{ml}$ ) [28]. In patients with fasting gastrin $<1,000 \mathrm{pg} / \mathrm{ml}$, the sensitivity of the secretin test using the criterion delta (increase from prestimulation level) gastrin of $\geq 110 \mathrm{pg} / \mathrm{ml}$ was $93 \%$ (CI 76-99\%) and for a delta gastrin of $200 \mathrm{pg} / \mathrm{ml}$ sensitivity was $85 \%$ (CI 66-96\%) ( $p>0.05)$ [28]. The same group recently reported their prospective experience on gastrin provocative tests in 293 patients from the NIH with ZES and compared with 537 ZES patients in the literature and 462 nonZES patients (again from the literature) [30]. This group established a delta gastrin of $\geq 120 \mathrm{pg} / \mathrm{ml}$ in patients with $<10$-fold increase as having the highest sensitivity and specificity (94 and 100\%, respectively) [30]. They also demonstrated the clear superiority of the secretin provocation test compared to the calcium test ( 94 vs. 62\%). However, in ZES patients with a negative secretin test the calcium provocation test may be helpful [30]. The expert group noted that certain groups had difficulty in obtaining secretin, hindering accurate diagnosis.

Indications for Gastrin Provocative Tests: Secretin Test - The secretin test is performed to confirm a biochemical diagnosis of gastrinoma. The test may be repeated during the follow-up after curative surgery. FSG should be performed prior to secretin test; if FSG $>1,000 \mathrm{pg} /$ $\mathrm{ml}$ a secretin test is not necessary. When FSG lies between 200 and $1,000 \mathrm{pg} / \mathrm{ml}$, a secretin test should be performed;

- The following conditions should also be documented:

$\circ$ Absence of fundic atrophic gastritis

- Antral and fundic biopsies ( \pm serology for antiparietal and intrinsic factor antibodies); 
- 24-hour pH-metry (loss of diurnal pH course); basal acid output is recommended pre and post secretin where possible; $\mathrm{BAO}>15 \mathrm{mmol} / \mathrm{h}$ is highly suggestive of diagnosis of ZES; a random $\mathrm{pH}$ analysis during upper gastrointestinal endoscopy was also suggested (this requires further evaluation);

- Helicobacter pylori testing;

- Other conditions leading to high FSG should be considered, including: gastric outlet obstruction, renal failure, antral G-cell syndromes, short bowel syndrome, retained gastric antrum.

\section{Preparation for Secretin Test}

- If possible, PPIs should be interrupted 10 days to 2 weeks prior to the test (PPIs for 2 weeks can be replaced by $\mathrm{H}_{2}$ blockers); interruption of $\mathrm{H}_{2}$ blockers for approximately $48 \mathrm{~h}$ prior to test, however, interruption of all anti-secretory medications should be individually adapted and patients should be warned of re-apparition of symptoms and should have sufficient antisecretory medications to start should they become symptomatic; certain patients may have to be hospitalized during anti-secretory therapy withdrawal;

- Heparinized vacutainers are used and should be labeled and placed in ice.

\section{Secretin Test}

- Patient fasting overnight, 12-14 h.

- Site indwelling i.v. cannula.

- Kabi-secretin (2 U/kg body weight) is given by intravenous bolus.

- Serum gastrin

o baseline measured at -15 , and -1 min before test;

$\circ 2,5,10,15,20$, and 30 min after secretin.

- Samples stored on ice (immediate transfer to laboratory).

Possible side effects of the secretin test include flush, allergic reaction.

\section{Interpretation of Results}

- Delta gastrin at least $200 \mathrm{pg} / \mathrm{ml}$ any time during the test is considered as positive.

- The NIH has recently published a delta gastrin of $\geq 120 \mathrm{pg} / \mathrm{ml}$ as having a high sensitivity and specificity (94 and 100\%, respectively) [30].

\section{Serum Chromogranin A}

Chromogranin $\mathrm{A}(\mathrm{Cg} \mathrm{A})$ is an acid glycoprotein with 439 amino acids that is present in the secretory dense core granules of most neuroendocrine cells [32]. The chromogranin family consists of at least three different watersoluble acidic glycoproteins ( $\mathrm{CgA}, \mathrm{CgB}$, and secretogranin II, sometimes called chromogranin C). Upon stimulation, CgA and other peptide hormones and neuropeptides are released. CgA is also secreted from neuroendocrine-derived tumors including foregut, midgut and hindgut gastrointestinal NETs, pheochromocytomas, neuroblastomas, medullary thyroid carcinomas, some pituitary tumors, functioning and non-functioning pancreatic NETs and other amine precursor uptake and decarboxylation tumors. CgA has also been widely used as an immunohistochemical marker in NETs [33] and is recognized as the most effective. CgA has been recognized as a general serum marker, as it is co-secreted in tumors with the amines and peptides that are present in the neurosecretory granules [34] and can be elevated in both functionally active and non-functional NETs. Specificity of elevated $\mathrm{CgA}$ is related to tumor type and is almost universally elevated in patients with gastrinoma [35-37]. It is often high in NETs from midgut origin and nonfunctioning pancreatic NETs. Differences in tumor cell type, histological differentiation and tumor volume may influence the level of $\mathrm{CgA}$ and interpretation may also depend on the assay used in measurement.

\section{Reliability of CgA in Patients with NETs}

Overall CgA has been found to be clinically informative and moderately sensitive in the majority of studies devoted to this topic. $\mathrm{CgA}$ was found to be more sensitive than neurone-specific enolase in all subgroups of a large mixed NET patient cohort $(\mathrm{n}=128)$ [38]. While performances have been limited in low-level cut-offs due to the overlap with control populations, very high levels of serum CgA are rarely found outside the setting of NETs with the exception of patients on gastric acid secretory blockers, especially PPIs (see below) [39] or those with hypergastrinaemia. Specificity of CgA in the diagnosis of NETs depends on the tumor type and burden $(100 \%$ specificities have been reported in patients with metastatic disease [40-43]), the quality of the control populations used and the cut-off values employed [36, 44]. Elevated CgA was found to be more sensitive than high urinary 5 HIAA levels in patients with metastatic midgut lesions (87 vs. 76\%, respectively) [5]. Nobels et al. [35] demonstrated a significant positive relation between the serum 
levels of CgA and the tumor mass in NETs; however, the distinction between high and low tumor volume may be open to question. This study also confirmed tissue specificities as high $\mathrm{CgA}$ concentrations were found in all patients with gastrinoma, although small in size and tumor volume [35]. In a mixed series of 128 patients with NET, increased $\mathrm{CgA}$ levels were found in 29 and $67 \%$ of patients with locoregional or metastatic disease, respectively [38]. Nonetheless, the prognostic value of $\mathrm{CgA}$ in patients with NET has not been confirmed to date.

False-positive elevation of CgA may occur in the following circumstances:

- Impaired renal function $[45,46]$;

- Parkinson disease, untreated hypertension and pregnancy;

- Steroid treatment or glucocorticoid excess, which can lead to up-regulation of CgA mRNA $[47,48]$;

- Chronic atrophic gastritis (type A) [49];

- Treatment with anti-secretory medications, especially PPIs [39].

Chronic elevation of gastrin levels provokes hyperplasia of the neuroendocrine cells of the stomach, and these cells are able to secrete $\mathrm{CgA}$ [50]. In patients with chronically elevated CgA and ZES, Stabile et al. [51] demonstrated that the CgA concentrations can be normalized by gastrectomy alone, without resection of the gastrinproducing tumor. A more recently described case report of false-positive $\mathrm{CgA}$ was due to the presence of heterophile antibodies ( $\mathrm{HAb}$ ), which can bind to animal antigens and may be present in up to $40 \%$ of the normal population [52]; in the $\mathrm{CgA}$ immunometric assays, $\mathrm{HAb}$ interferences may be circumnavigated by using a HAbblocking tube [53].

\section{Assays for $C g A$}

A recognized international standard for $\mathrm{CgA}$ assay is not available and variations in assay types may influence results. Several assays for measurements of intact $\mathrm{CgA}$ and cleavage products have been developed $[34,54]$. The complexity of assays is explained by the presence of several CgA-related peptides from human and other species [55] and CgA processing varies according to neuroendocrine cells/tissue [56, 57]. A competitive radioimmunoassay can detect circulating $\mathrm{CgA}$, with the use of purified full-length human CgA $[41,58]$. Commercial CgA kits differ in the types of antibodies used (monoclonal vs. polyclonal) and include enzyme detection (ELISA) and radioimmunoassay. Differences in methods of standardization have also led to heterogeneity. Generally, measurement of intact CgA in plasma has greater sensitivity for the diagnosis of NET than the measurement of fragments $[34,59]$. Stridsberg et al. [44] compared the three commercially available kits in a group of NET patients and found sensitivities to vary between 67 and 93\%, while specificities were $>85 \%$ for all three. A recent multicentre prospective comparison between two methods, immunoradiometric and ELISA, found a 36\% clinical discordance rate [60]. These results were mirrored with a difference of 5-fold inter-laboratory variation rate in a recent Italian study aimed at assessing $\mathrm{CgA}$ detection performance as applied to immunoradiometric and ELISA assays [61]. A further prospective analysis underlined $\mathrm{CgA}$ to be a practical marker in patients with NET, however, with limited diagnostic power; using RCO curves, a cut-off of $53 \mathrm{ng} / \mathrm{ml}$ for IRMA and 16 U/l for ELISA for discriminating between healthy controls and NET patients yielded only moderate sensitivities (71.3 and 83\%, respectively) and specificities (71 and $85 \%$, respectively) [43].

\section{General Remarks on CgA}

- CgA is the most practical and useful general serum tumor marker in patients with NET;

- Elevated CgA can occur in normal individuals and in patients with non-NET tumors although the levels are usually lower than in patients with NET;

- Sensitivity of elevated CgA varies according to NET tumor type and volume.

\section{CgA Assays and Interpretation}

- Reference laboratories should be preferred for clinical samples assays;

- Reference intervals and individual patient results differ significantly between different chromogranin A assays and cannot be directly compared;

- Serial measurements should be performed using the same assay;

- If assays are changed, patients should undergo a new baseline measurement;

- False-positive results are possible in patients with hypergastrinaemia (especially on anti-secretory medications or chronic atrophic gastritis type A) and in the presence of heterophile antibodies (care in patients autoimmune disease or those sensitized to rodent proteins (mouse monoclonal antibodies));

- Where possible, proton pump inhibitors should be interrupted, leaving a clearance of at least 3 half-lives, prior to CgA plasma sampling. 


\section{List of Participants}

List of Participants of the Consensus Conference on the ENETS Guidelines for the Standard of Care for the Diagnosis and Treatment of Neuroendocrine Tumors, Held in Palma de Mallorca (Spain), November 28 to December 1, 2007

Göran Åkerström, Department of Surgery, University Hospital, Uppsala (Sweden); Bruno Annibale, University Sapienza Roma, Rome (Italy); Rudolf Arnold, Department of Internal Medicine, Philipps University, Munich (Germany); Emilio Bajetta, Medical Oncology Unit B, Istituto Nazionale Tumori, Milan (Italy); Yuan-Jia Chen, Department of Gastroenterology, Peking Union Medical College Hospital, Chinese Academy of Medical Sciences, Beijing (China); Frederico Costa, Hospital Sirio Libanes, Centro de Oncologia, São Paulo (Brazil); Anne Couvelard, Service de Gastroentérologie, Hôpital Beaujon, Clichy (France); Joseph Davar, Department of Cardiology, Royal Free Hospital, London (UK); Wouter de Herder, Department of Internal Medicine, Section of Endocrinology, Erasmus MC, Rotterdam (The Netherlands); Barbro Eriksson, Medical Department, Endocrine Unit, University Hospital, Uppsala (Sweden); Massimo Falconi, Medicine and Surgery, University of Verona, Verona (Italy); Diego Ferone, Departments of Internal Medicine and Endocrinological and Metabolic Sciences, University of Genoa, Genoa (Italy); Björn Gustafsson, Medisinsk avd, Gastroseksjon, St Olavs Hospital, Trondheim (Norway); Rudolf Hyrdel, II. Internal Medical Department, University Hospital Martin, Martin (Slovakia); Diana Ivan, Endocrinology and Diabetology, Klinikum der Philipps-Universität, Marburg (Germany); Gregory Kaltsas, G. Genimatas Hospital, Athens (Greece); Reza Kianmanesh, UFR Bichat-Beaujon-Louis Mourier, Service de Chirurgie Digestive, Hôpital Louis Mourier, Colombes (France); Günter Klöppel, Institut für Pathologie, TU München, Munich (Germany); UlrichPeter Knigge, Department of Surgery, Rigshospitalet, Copenhagen (Denmark); Paul Komminoth, Institute for Pathology, Stadtspital Triemli, Zürich (Switzerland); Beata Kos-Kudła, Slaska Akademia Medyczna Klinika Endokrynologii, Zabrze (Poland);
Dik Kwekkeboom, Department of Nuclear Medicine, Erasmus University Medical Center, Rotterdam (The Netherlands); $R a$ chida Lebtahi, Nuclear Medicine Department, Bichat Hospital, Paris (France); Val Lewington, Royal Marsden, NHS Foundation Trust, Sutton (UK); Anne Marie McNicol, Division of Cancer Sciences and Molecular Pathology, Pathology Department, Royal Infirmary, Glasgow (UK); Emmanuel Mitry, Hepatogastroenterology and Digestive Oncology, Hôpital Ambroise-Paré, Boulogne (France); Ola Nilsson, Department of Pathology, Sahlgrenska sjukhuset, Gothenburg (Sweden); Kjell Öberg, Department of Internal Medicine, Endocrine Unit, University Hospital, Uppsala (Sweden); Mauro Papotti, Department of Biological and Clinical Sciences, University of Turin/St. Luigi Hospital, Turin (Italy); Marianne Pavel, Department of Hepatology and Gastroenterology, Campus Virchow-Klinikum, Charité-Universitätsmedizin Berlin, Berlin (Germany); Aurel Perren, Institut für Allgemeine Pathologie und Pathologische Anatomie der Technischen Universität München, Klinikum r.d. Isar, Munich (Germany); Marco Platania, Istituto Nazionale dei Tumori di Milano, Milan (Italy); Guido Rindi, Department of Pathology and Laboratory Medicine, Università degli Studi, Parma (Italy); Philippe Ruszniewski, Service de Gastroentérologie, Hôpital Beaujon, Clichy (France); Ramon Salazar, Institut Català d'Oncologia, Barcelona (Spain); Aldo Scarpa, Department of Pathology, University of Verona, Verona (Italy); Klemens Scheidhauer, Klinikum rechts der Isar, TU München, Munich (Germany); Jean-Yves Scoazec, Anatomie Pathologique, Hôpital Edouard-Herriot, Lyon (France); Anders Sundin, Department of Radiology, Uppsala University Hospital, Uppsala (Sweden); Waldemar Szpak, Westville Hospital, Mayville (South Africa); Babs Taal, Netherlands Cancer Centre, Amsterdam (The Netherlands); Pavel Vitek, Institute of Radiation Oncology, University Hospital, Prague (Czech Republic); Marie-Pierre Vullierme, Service de Gastroentérologie, Hôpital Beaujon, Clichy (France); Bertram Wiedenmann, Department of Internal Medicine, Division of Hepatology and Gastroenterology, Campus Virchow-Klinikum, Charité-Universitätsmedizin Berlin, Berlin (Germany).

\section{References}

1 Meijer WG, Kema IP, Volmer M, Willemse $\mathrm{PH}$, de Vries EG: Discriminating capacity of indole markers in the diagnosis of carcinoid tumors. Clin Chem 2000;46:1588-1596.

2 Feldman JM: Urinary serotonin in the diagnosis of carcinoid tumors. Clin Chem 1986; 32:840-844.

-3 Baudin E, Bidart JM, Rougier P, et al: Screening for multiple endocrine neoplasia type 1 and hormonal production in apparently sporadic neuroendocrine tumors. J Clin Endocrinol Metab 1999;84:69-75.

4 Feldman JM, O'Dorisio TM: Role of neuropeptides and serotonin in the diagnosis of carcinoid tumors. Am J Med 1986;81:41-48.
5 Janson ET, Holmberg L, Stridsberg M, et al: Carcinoid tumors: analysis of prognostic factors and survival in 301 patients from a referral center. Ann Oncol 1997;8:685-690.

-6 Turner GB, Johnston BT, McCance DR, et al: Circulating markers of prognosis and response to treatment in patients with midgut carcinoid tumours. Gut 2006;55:1586-1591.

7 Van der Horst-Schrivers AN, Post WJ, Kema IP, et al: Persistent low urinary excretion of 5 -HIAA is a marker for favourable survival during follow-up in patients with disseminated midgut carcinoid tumours. Eur J Cancer 2007;43:2651-2657.
8 Formica V, Wotherspoon A, Cunningham $\mathrm{D}$, et al: The prognostic role of WHO classification, urinary 5-hydroxyindoleacetic acid and liver function tests in metastatic neuroendocrine carcinomas of the gastroenteropancreatic tract. Br J Cancer 2007;96:11781182.

$\checkmark 9$ Kissinger PT, Felice LJ, Riggin RM, Pachla LA, Wenke DC: Electrochemical detection of selected organic components in the eluate from high-performance liquid chromatography. Clin Chem 1974;20:992-997.

10 Mailman RB, Kilts CD: Analytical considerations for quantitative determination of serotonin and its metabolically related products in biological matrices. Clin Chem 1985; 31:1849-1854.
O’Toole/Grossman/Gross/Delle Fave/ Barkmanova/O'Connor/Pape/Plöckinger 
-11 Kema IP, de Vries EG, Muskiet FA: Clinical chemistry of serotonin and metabolites. J Chromatogr B Biomed Sci Appl 2000;747: 33-48.

$\checkmark 12$ Kroll CA, Magera MJ, Helgeson JK, Matern D, Rinaldo P: Liquid chromatographic-tandem mass spectrometric method for the determination of 5-hydroxyindole-3-acetic acid in urine. Clin Chem 2002;48:20492051.

13 Kema IP, Meijer WG, Meiborg G, Ooms B, Willemse PH, de Vries EG: Profiling of tryptophan-related plasma indoles in patients with carcinoid tumors by automated, online, solid-phase extraction and HPLC with fluorescence detection. Clin Chem 2001;47: 1811-1820.

-14 Mulder EJ, Oosterloo-Duinkerken A, Anderson GM, De Vries EG, Minderaa RB, Kema IP: Automated on-line solid-phase extraction coupled with HPLC for measurement of 5-hydroxyindole-3-acetic acid in urine. Clin Chem 2005;51:1698-1703.

$\checkmark 15$ Cleare AJ, Keating J, Ealing J, Sherwood RA: A case of coeliac disease detected via raised 5-hydroxytryptamine and 5-hydroxyindoleacetic acid. Ann Clin Biochem 1997;34: 440-441.

16 Bearcroft CP, Perrett D, Farthing MJ: Postprandial plasma 5-hydroxytryptamine in diarrhoea predominant irritable bowel syndrome: a pilot study. Gut 1998;42:42-46.

$\checkmark 17$ Nuttall KL, Pingree SS: The incidence of elevations in urine 5-hydroxyindoleacetic acid. Ann Clin Lab Sci 1998;28:167-174.

18 Kema IP, Schellings AM, Meiborg G, Hoppenbrouwers CJ, Muskiet FA: Influence of a serotonin- and dopamine-rich diet on platelet serotonin content and urinary excretion of biogenic amines and their metabolites. Clin Chem 1992;38:1730-1736.

-19 Mashige F, Matsushima Y, Kanazawa H, et al: Acidic catecholamine metabolites and 5hydroxyindoleacetic acid in urine: the influence of diet. Ann Clin Biochem 1996;33:4349.

20 Service FJ: Hypoglycemic disorders. N Engl J Med 1995;332:1144-1152.

-21 Hirshberg B, Skarulis MC, Pucino F, Csako G, Brennan R, Gorden P: Repaglinide-induced factitious hypoglycemia. J Clin Endocrinol Metab 2001;86:475-477.

-22 De Herder WW, Niederle B, Scoazec JY, et al: Well-differentiated pancreatic tumor/carcinoma: insulinoma. Neuroendocrinology 2006;84:183-188.

-23 Service FJ, Dale AJ, Elveback LR, Jiang NS: Insulinoma: clinical and diagnostic features of 60 consecutive cases. Mayo Clin Proc 1976;51:417-429.

24 Hirshberg B, Livi A, Bartlett DL, et al: Fortyeight-hour fast: the diagnostic test for insulinoma. J Clin Endocrinol Metab 2000;85: 3222-3226.
5 Service FJ, Natt N: The prolonged fast. J Clin Endocrinol Metab 2000;85:3973-3974.

26 Grant CS: Insulinoma. Best Pract Res Clin Gastroenterol 2005;19:783-798.

27 Berna MJ, Hoffmann KM, Serrano J, Gibril F, Jensen RT: Serum gastrin in Zollinger-Ellison syndrome: I. Prospective study of fasting serum gastrin in 309 patients from the National Institutes of Health and comparison with 2,229 cases from the literature. Medicine (Baltimore) 2006;85:295-330.

28 Frucht H, Howard JM, Slaff JI, et al: Secretin and calcium provocative tests in the Zollinger-Ellison syndrome. A prospective study. Ann Intern Med 1989;111:713-722.

29 Frucht H, Howard JM, Stark HA, et al: Prospective study of the standard meal provocative test in Zollinger-Ellison syndrome. Am J Med 1989;87:528-536.

-30 Berna MJ, Hoffmann KM, Long SH, Serrano J, Gibril F, Jensen RT: Serum gastrin in Zollinger-Ellison syndrome. II. Prospective study of gastrin provocative testing in 293 patients from the National Institutes of Health and comparison with 537 cases from the literature. Evaluation of diagnostic criteria, proposal of new criteria, and correlations with clinical and tumoral features. Medicine (Baltimore) 2006;85:331-364.

31 Jensen RT, Niederle B, Mitry E, et al: Gastrinoma (duodenal and pancreatic). Neuroendocrinology 2006;84:173-182.

32 Deftos LJ: Chromogranin A: its role in endocrine function and as an endocrine and neuroendocrine tumor marker. Endocr Rev 1991;12:181-187.

33 Rindi G, Kloppel G: Endocrine tumors of the gut and pancreas tumor biology and classification. Neuroendocrinology 2004;80(suppl 1):12-15.

34 O'Connor DT, Deftos LJ: Secretion of chromogranin A by peptide-producing endocrine neoplasms. N Engl J Med 1986;314: 1145-1151.

- 35 Nobels FR, Kwekkeboom DJ, Coopmans W, et al: Chromogranin A as serum marker for neuroendocrine neoplasia: comparison with neuron-specific enolase and the $\alpha$-subunit of glycoprotein hormones. J Clin Endocrinol Metab 1997;82:2622-2628.

36 Taupenot L, Harper KL, O’Connor DT: The chromogranin-secretogranin family. N Engl J Med 2003;348:1134-1149.

- 37 Goebel SU, Serrano J, Yu F, Gibril F, Venzon DJ, Jensen RT: Prospective study of the value of serum chromogranin A or serum gastrin levels in the assessment of the presence, extent, or growth of gastrinomas. Cancer 1999; 85:1470-1483.

38 Baudin E, Gigliotti A, Ducreux M, et al: Neuron-specific enolase and chromogranin $\mathrm{A}$ as markers of neuroendocrine tumours. Br J Cancer 1998;78:1102-1107.
39 Sanduleanu S, De Bruine A, Stridsberg M, et al: Serum chromogranin A as a screening test for gastric enterochromaffin-like cell hyperplasia during acid-suppressive therapy. Eur J Clin Invest 2001;31:802-811.

40 Sobol RE, Memoli V, Deftos LJ: Hormonenegative, chromogranin A-positive endocrine tumors. N Engl J Med 1989;320:444-447.

41 O'Connor DT, Pandlan MR, Carlton E, Cervenka JH, Hslao RJ: Rapid radioimmunoassay of circulating chromogranin A: in vitro stability, exploration of the neuroendocrine character of neoplasia, and assessment of the effects of organ failure. Clin Chem 1989;35: 1631-1637.

42 Eriksson B, Arnberg H, Oberg K, et al: A polyclonal antiserum against chromogranin $\mathrm{A}$ and $\mathrm{B}-\mathrm{a}$ new sensitive marker for neuroendocrine tumours. Acta Endocrinol (Copenh) 1990;122:145-155.

43 Zatelli MC, Torta M, Leon A, et al: Chromogranin $A$ as a marker of neuroendocrine neoplasia: an Italian Multicenter Study. Endocr Relat Cancer 2007;14:473-482.

44 Stridsberg M, Eriksson B, Oberg K, Janson ET: A comparison between three commercial kits for chromogranin A measurements. J Endocrinol 2003;177:337-341.

-45 Hsiao RJ, Mezger MS, O'Connor DT: Chromogranin A in uremia: progressive retention of immunoreactive fragments. Kidney Int 1990;37:955-964.

-46 Stridsberg M, Husebye ES: Chromogranin A and chromogranin B are sensitive circulating markers for phaeochromocytoma. Eur J Endocrinol 1997;136:67-73.

47 Rozansky DJ, Wu H, Tang K, Parmer RJ, O'Connor DT: Glucocorticoid activation of chromogranin A gene expression. Identification and characterization of a novel glucocorticoid response element. J Clin Invest 1994;94:2357-2368.

48 Giovanella L, La Rosa S, Ceriani L, Uccella S, Erba P, Garancini S: Chromogranin-A as a serum marker for neuroendocrine tumors: comparison with neuron-specific enolase and correlation with immunohistochemical findings. Int J Biol Markers 1999;14:160-166.

49 O'Toole D: Current trend: endocrine tumors of the stomach, small bowel, colon and rectum (in French). Gastroenterol Clin Biol 2006;30:276-291.

50 D'Adda T, Corleto V, Pilato FP, et al: Quantitative ultrastructure of endocrine cells of oxyntic mucosa in Zollinger-Ellison syndrome. Correspondence with light microscopic findings. Gastroenterology 1990;99:17-26.

- 51 Stabile BE, Howard TJ, Passaro E Jr, O'Connor DT: Source of plasma chromogranin A elevation in gastrinoma patients. Arch Surg 1990;125:451-453.

\$2 Levinson SS, Miller JJ: Towards a better understanding of heterophile (and the like) antibody interference with modern immunoassays. Clin Chim Acta 2002;325:1-15. 
53 Giovanella L, Ceriani L, Lumastro C, Ghelfo A: False-positive serum chromogranin A assay due to heterophile antibody interference. Clin Chim Acta 2007;379:171-172.

54 Stridsberg M: Measurements of chromogranins and chromogranin-related peptides by immunological methods. Adv Exp Med Biol 2000;482:319-327.

55 Laslop A, Doblinger A, Weiss U: Proteolytic processing of chromogranins. Adv Exp Med Biol 2000;482:155-166.

56 Portela-Gomes GM, Stridsberg M: Selective processing of chromogranin A in the different islet cells in human pancreas. J Histochem Cytochem 2001;49:483-490.
Iacangelo AL, Eiden LE: Chromogranin A: current status as a precursor for bioactive peptides and a granulogenic/sorting factor in the regulated secretory pathway. Regul Pept 1995;58:65-88.

58 O'Connor DT, Bernstein KN: Radioimmunoassay of chromogranin A in plasma as a measure of exocytotic sympathoadrenal activity in normal subjects and patients with pheochromocytoma. N Engl J Med 1984;311: 764-770.

59 Stridsberg M, Oberg K, Li Q, Engstrom U, Lundqvist G: Measurements of chromogranin A, chromogranin B (secretogranin I), chromogranin $\mathrm{C}$ (secretogranin II) and pancreastatin in plasma and urine from patients with carcinoid tumours and endocrine pancreatic tumours. J Endocrinol 1995;144:4959.
60 Leon A, Torta M, Dittadi R, et al: Comparison between two methods in the determination of circulating chromogranin A in neuroendocrine tumors: results of a prospective multicenter observational study. Int J Biol Markers 2005;20:156-168.

61 Verderio P, Dittadi R, Marubini E, et al: An Italian program of external quality control for chromogranin A ( $\mathrm{CgA})$ assay: performance evaluation of $\mathrm{CgA}$ determination. Clin Chem Lab Med 2007;45:1244-1250. 